\title{
DETERMINAÇÃO DO ÍNDICE DE TEMPERATURA E UMIDADE (ITU) PARA PRODUÇÃO DE AVES NA MESORREGIÃO METROPOLITANA DE CURITIBA - PR
}

\author{
Determination of the Temperature and Humidity Index (ITU) \\ for the Birds Production in Metropolitan Mesoregion \\ of Curitiba - PR
}

\author{
Eduardo Teixeira da Silva ${ }^{1}$ \\ Daniel Guedes Leite ${ }^{2}$ \\ Flavio Manabu Yuri ${ }^{2}$ \\ Francine da Silva G. Nery ${ }^{2}$ \\ Josiane Crystina Costa Rego ${ }^{2}$ \\ Roberta de Azevedo Zanatta ${ }^{2}$ \\ Samuel Augusto dos Santos² \\ Vinicius Vieira Moura ${ }^{2}$
}

\section{Resumo}

Objetivou-se, com este trabalho, determinar o Índice de Temperatura e Umidade (ITU) para a produção de aves na mesorregião Metropolitana de Curitiba, como orientação a avicultores e futuros avicultores na implantação de sistemas de controle ambiental. A determinação do ITU foi realizada com os dados climáticos fornecidos pelo IAPAR (normais climatológicas) das seguintes microrregiões: Antonina, Cerro Azul, Guaraqueçaba, Lapa, Morretes e Pinhais. Os valores da Temperatura Média do Ar Compensada, Umidade Relativa e ITU foram utilizados para comparar com as condições de conforto térmico para aves, em função da idade. As comparações realizadas em cada microrregião mostraram a necessidade de corrigir o microclima dos galpões para alcançar condições ideais para produção de aves.

Palavras-chave: Conforto térmico; Clima; Avicultura.

\section{Abstract}

The main objective of this work was to determine the Temperature and Humidity Index (ITU) for the poultry production in the Metropolitan region of Curitiba, as guidance to actual and future poultry producers for the implantation of environmental control system. The determination of ITU was accomplished with the climatic data supplied by IAPAR (climatologic normal) of the following regions: Antonina, Cerro Azul, Guaraqueçaba, Lapa, Morretes and Pinhais. The values of the Air Compensated Temperature Average, Relative Humidity and ITU were used to compare with the conditions of thermal comfort for poultry, as function of age. The comparisons accomplished in each region showed the need to correct the micro-climate of the shed to reach ideal conditions for poultry production.

Keywords: Thermal comfort; Climate; Poultry.

1 Engenheiro Agrícola, Doutor, UFPR, Depto de Solos e Eng. Agrícola, Prof. Adjunto, R. dos Funcionários, 1540 CEP 80035-050, Curitiba, PR. Eduardo@ufpr.br

2 Alunos do Curso de Zootecnia - Universidade Federal do Paraná - SCA - Estagiários Departamento de Solos e Engenharia Agrícola. 


\section{Introdução}

A avicultura brasileira registrou, nos últimos anos, aumentos significativos na produção de carne, ultrapassando 8,04 milhões de toneladas em 2003, o que mantém o Brasil como o segundo maior produtor mundial. Contudo, em muitas regiões brasileiras, a radiação solar intensa conduz a altos valores de temperatura do ar, principalmente no verão, gerando condições de desconforto térmico ao bom desempenho avícola, nas suas variadas formas de exploração.

O controle do ambiente para criação de aves tem papel relevante, uma vez que pesquisas têm comprovado a importância dos fatores climáticos sobre a produção destes animais, segundo Piasentini (1984).

O mecanismo de homeostase, entretanto, é eficiente somente quando a temperatura ambiente está dentro de certos limites, segundo Tinoco (1998). Portanto, é importante que as instalações sejam mantidas a temperaturas próximas às das condições de conforto. Outro fator importante, que interage juntamente com a temperatura, é a umidade relativa do ar. A umidade relativa em ambientes é limitante, para a maioria dos homeotermos adulto, quando a temperatura supera a marca dos $24^{\circ} \mathrm{C}$ e mais pronunciadamente quando ultrapassa o valor de $30^{\circ} \mathrm{C}$, segundo Muller (1982). A umidade relativa passa a ter importância no conforto térmico das aves quando a temperatura ambiental atinge $25^{\circ} \mathrm{C}$. Altas taxas de umidade relativa, associadas a temperaturas altas, fazem com que menos umidade seja removida das vias aéreas, tornando a respiração cada vez mais ofegante, segundo Tinoco (1998). Sendo assim, a inter-relação entre as características climáticas regionais com as exigências bioclimáticas das aves será de grande importância no melhoramento e aumento do rendimento da produção de monogástricos na mesorregião.

Portanto, construir instalações adequadas ao clima e que permitam a manutenção de temperatura, umidade relativa, velocidade do ar, em limites que proporcionam ambiente ideal no interior do galpão de acordo com as exigências das aves, sem aumento dos custos de produção, tem sido grande desafio. $\mathrm{O}$ objetivo deste trabalho foi de determinar o Índice de Temperatura e Umidade (ITU) para a produção de aves na Mesorregião da Região Metropolitana de Curitiba como orientação aos avicultores e futuros avicultores na implantação de sistemas de controle ambiental.

\section{Material e métodos}

O Estado do Paraná localiza-se na Região Sul do País, ocupando uma área de $199.725 \mathrm{~km}^{2}$, que corresponde a 2,3\% da superfície total do Brasil, situando-se entre os meridianos de $48^{\circ} 30^{\prime}$ e $54^{\circ} 30^{\prime}$, de longitude a oeste de Greenwich e os paralelos de $22^{\circ} 30^{\prime}$ e $26^{\circ} 30^{\prime}$ de latitude sul. O Paraná está dividido em 10 mesorregiões e, para este trabalho, foi utilizada a mesorregião: Metropolitana de Curitiba. Foram selecionadas seis estações agrometeorológicas, uma em cada uma das seguintes microrregiões: Antonina, Cerro Azul, Guaraqueçaba, Lapa, Morretes e Pinhais. O quadro 01 abaixo apresenta caracterização das microrregiões. A determinação do ITU foi realizada com os dados climáticos obtidos das normais climatológicas fornecidos pelo (Instituto Agronômico do Paraná - Área de Ecofisiologia) IAPAR.

\section{Quadro 01 - Caracterização das microrregiões analisadas.}

Chart 01 - Characterization of the analyzed microregions.

\begin{tabular}{|l|l|l|l|l|l|l|}
\hline \multicolumn{9}{|c|}{} & \multicolumn{2}{c|}{ Dados climáticos } \\
\hline Microrregião & Longitude & Latitude & Altitude (m) & Área $\mathbf{( k m}^{2}$ ) & \multicolumn{1}{|c|}{ Ano início } & Ano fim \\
\hline Antonina & $25^{\circ} 13^{\prime} \mathrm{S}$ & $48^{\circ} 48^{\prime} \mathrm{W}$ & 60 & 845,853 & 1987 & 1999 \\
\hline Cerro Azul & $24^{\circ} 49^{\prime} \mathrm{S}$ & $59^{\circ} 15^{\prime} \mathrm{W}$ & 360 & $1.153,238$ & 1987 & 1998 \\
\hline Guaraqueçaba & $25^{\circ} 16^{\prime} \mathrm{S}$ & $48^{\circ} 32^{\prime} \mathrm{W}$ & 40 & $2.317,024$ & 1987 & 2003 \\
\hline Lapa & $25^{\circ} 47^{\prime} \mathrm{S}$ & $49^{\circ} 46^{\prime} \mathrm{W}$ & 910 & $2.145,352$ & 1988 & 2004 \\
\hline Morretes & $25^{\circ} 30^{\prime} \mathrm{S}$ & $48^{\circ} 49^{\prime} \mathrm{W}$ & 59 & 662,758 & 1987 & 2003 \\
\hline Pinhais & $25^{\circ} 25^{\prime} \mathrm{S}$ & $49^{\circ} 46^{\prime} \mathrm{W}$ & 930 & 60,796 & 1987 & 1998 \\
\hline
\end{tabular}


tes variáveis:

Para o estudo, foram utilizadas as seguin-

- Média da Temperatura do Ar Compensada (t med);

- Umidade Relativa do Ar (UR); e

- Índice de temperatura e Umidade (ITU).

O Índice de Temperatura e Umidade (ITU)

foi determinado pela equação (01) abaixo, desenvolvida por Thom (1958):

$$
\begin{aligned}
& \text { ITU }=T_{b s}+0,36 T_{p o}-330,08 \\
& \text { Onde: } \\
& T_{b s}=\text { temperatura de bulbo seco }(K)
\end{aligned}
$$

$\mathrm{T}_{\mathrm{po}}=$ temperatura de ponto de orvalho

Estes valores foram utilizados para comparar com as condições de conforto térmico ideais para aves, em função da sua idade em semanas de vida (tabela 1). Para comparar as exigências das aves com os valores climáticos das microrregiões, foi adotada a seguinte simbologia:

I - Inferiores aos exigidos pelas aves;

C - confortáveis aos exigidos pelas aves e;

$$
\text { S - superiores aos exigidos pelas aves. }
$$

\section{Tabela 1 - Valores ideais de temperatura, umidade relativa do ar e do índice de temperatura} e umidade (ITU), em função da idade das aves.

Table 1 - Temperature optimal values, air relative humidity and temperature and humidity index (ITU), as function of the bird ages.

\begin{tabular}{l|l|l|l}
\hline Idade (Semanas) & Temperatura ( $\left.{ }^{\circ} \mathbf{C}\right)$ & Umidade Relativa do Ar (\%) & ITU ideal \\
\hline 1 & $32-35$ & $60-70$ & $72,4-80$ \\
2 & $29-32$ & $60-70$ & $68,4-76$ \\
3 & $26-29$ & $60-70$ & $64,5-72$ \\
4 & $23-26$ & $60-70$ & $60,5-68$ \\
5 & $20-23$ & $60-70$ & $56,6-64$ \\
6 & 20 & $60-70$ & $56,6-60$ \\
7 & 20 & $60-70$ & $56,6-60$ \\
\hline
\end{tabular}

\section{Resultados e discussão}

\section{- Município de Antonina}

Analisando as tabelas do ITU (TABELAS 1 e 2), meses de janeiro a dezembro, referente ao ciclo de vida de aves de corte, verificou-se que para a primeira semana de idade o valor encontrado no município Antonina, para os meses entre janeiro $(73,88)$ a fevereiro $(73,59)$, esses resultaram em valores dentro da faixa de conforto térmico para aves nesta idade (ITU ideal 72,4 - 80), necessitando de aquecimento do ambiente a partir do mês de março $(72,52)$ até dezembro $(72,58)$, o que pode ser observado pela Figura 1. Para Segunda semana de idade (ITU ideal 68,5 - 76), durante os meses de novembro $(70,36)$ a abril $(70,28)$, verificou-se que os valores se localizaram dentro da faixa de conforto térmico, necessitando, portanto, de aquecimento entre os meses de maio $(66,04)$ a outubro $(67,72)$. Para a terceira semana de idade (ITU ideal 64,5 - 72), durante os meses de dezembro $(72,58)$ a março $(72,52)$, verificou-se a necessidade de um sistema de resfriamento, pois, durante esses meses, os valores excederam o limite superior da faixa de conforto térmico para aves nesta idade; porém, entre os meses de junho $(63,35)$ a agosto $(63,22)$, necessitou-se de um sistema de aquecimento, pois os valores mostraram-se abaixo da faixa de conforto térmico para aves nesta idade. Os meses de abril, maio, setembro, outubro e novembro apresentaram valores dentro da faixa de conforto térmico. Para a quarta semana de idade (ITU ideal 56,6 - 64), nos meses entre setembro $(65,06)$ a maio $(66,04)$, necessitou-se de sistema de resfriamento do ambiente, pois apresentaram valores superiores ao máximo ideal, enquanto que, durante os meses de junho a $(63,35)$ a agosto $(63,22)$, os valores encontraram-se dentro da faixa de conforto térmico para aves. Para sexta semana de idade (ITU 56,6 - 60), pôde-se verificar que durante todos os meses do ano, o município apresentou valores superiores ao máximo recomendado para aves, necessitando, portanto, de sistema de resfriamento do ambiente, o que pode ser observado na Figura 2. 
Tabela 2 - Índice de temperatura e umidade (ITU) para o município de Antonina.

Table 2 - Temperature and humidity index (ITU) of Antonina city

\begin{tabular}{|c|c|c|c|c|c|c|}
\hline & \multicolumn{6}{|c|}{ ITU } \\
\hline & \multicolumn{6}{|c|}{ Idade das Aves em Semanas } \\
\hline Meses do ano & 1 & 2 & 3 & 4 & 5 & 6 \\
\hline Janeiro & I & $\mathrm{C}$ & $\mathrm{S}$ & $\mathrm{S}$ & $\mathrm{S}$ & $\mathrm{S}$ \\
\hline Fevereiro & I & $\mathrm{C}$ & $\mathrm{S}$ & $\mathrm{S}$ & $\mathrm{S}$ & $\mathrm{S}$ \\
\hline Março & I & $\mathrm{C}$ & $\mathrm{S}$ & $\mathrm{S}$ & $\mathrm{S}$ & $\mathrm{S}$ \\
\hline Abril & I & $\mathrm{C}$ & $\mathrm{C}$ & $\mathrm{S}$ & $\mathrm{S}$ & $\mathrm{S}$ \\
\hline Maio & I & I & $\mathrm{C}$ & $\mathrm{C}$ & S & S \\
\hline Junho & I & I & I & $\mathrm{C}$ & $\mathrm{C}$ & $\mathrm{S}$ \\
\hline Julho & I & I & I & $\mathrm{C}$ & $\mathrm{C}$ & $\mathrm{S}$ \\
\hline Agosto & I & I & $\mathrm{C}$ & $\mathrm{C}$ & $\mathrm{C}$ & $\mathrm{s}$ \\
\hline Setembro & I & I & $\mathrm{C}$ & $\mathrm{C}$ & $\mathrm{S}$ & $\mathrm{S}$ \\
\hline Outubro & I & $\mathrm{C}$ & $\mathrm{C}$ & $\mathrm{C}$ & $\mathrm{S}$ & $\mathrm{S}$ \\
\hline Novembro & I & $\mathrm{C}$ & $\mathrm{C}$ & $\mathrm{s}$ & $\mathrm{S}$ & $\mathrm{S}$ \\
\hline Dezembro & I & $\mathrm{C}$ & $\mathrm{S}$ & $\mathrm{S}$ & $\mathrm{S}$ & $\mathrm{S}$ \\
\hline
\end{tabular}

Figura 1 - Gráfico ITU obtido para aves na $1^{\text {a }}$ semana de idade em Antonina. Figure 1 - ITU Graph for birds at first week of age in Antonina.

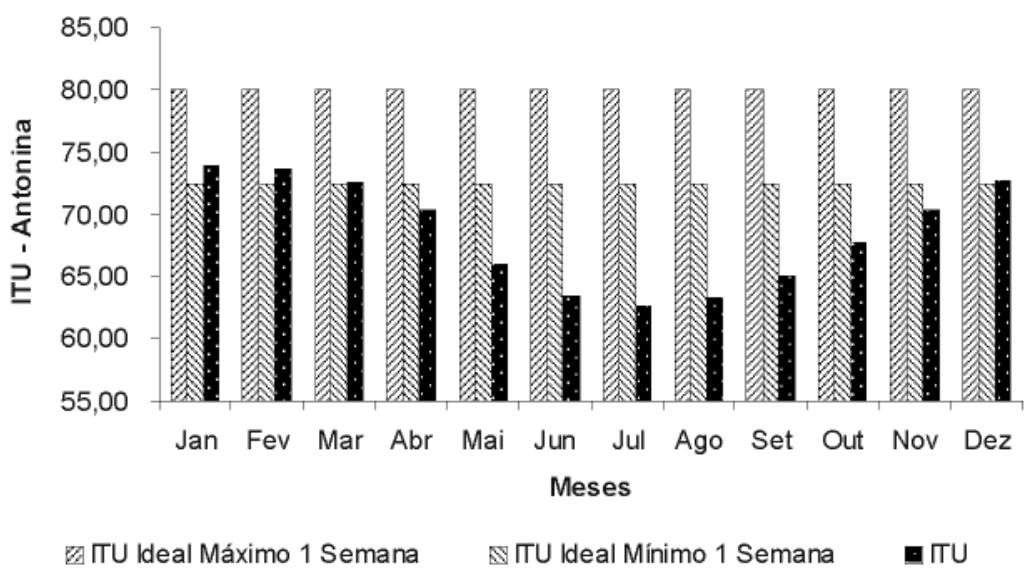




\section{Figura 2 - Gráfico ITU obtido para aves na $\boldsymbol{6}^{\mathrm{a}}$ semana de idade em Antonina.}

Figure 2 - ITU Graph for birds at sixth week of age in Antonina

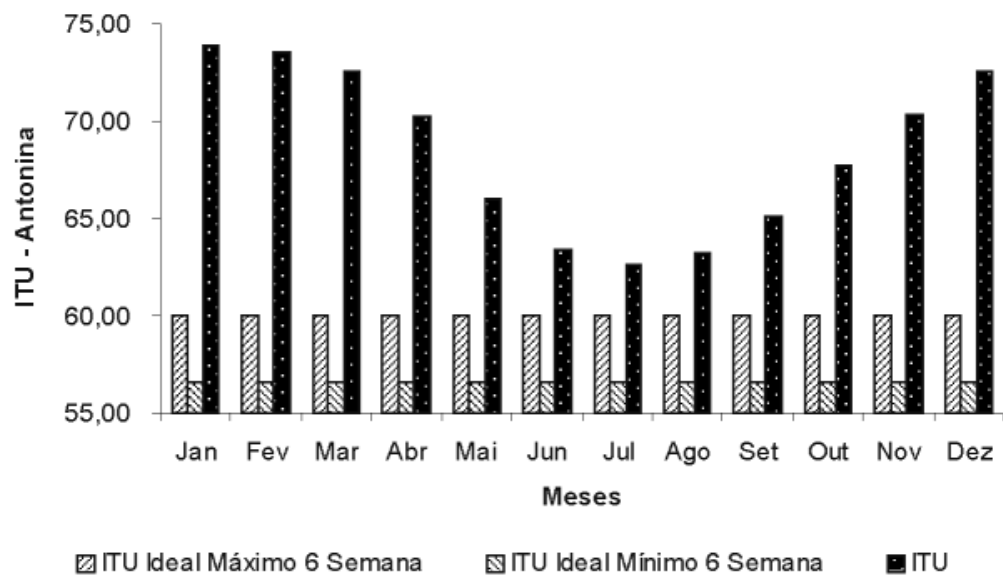

\section{- Município Cerro Azul}

Analisando as tabelas do ITU (TABELAS 1 e 3), durante os meses de janeiro a dezembro, referente ao ciclo de vida de aves de corte, verificou-se que, para a primeira semana de idade, 0 valor encontrado no município Cerro Azul, para os meses entre dezembro e fevereiro $(73,20)$, esses resultaram em valores dentro da faixa de conforto térmico para aves nesta idade, necessitando de aquecimento entre os meses de março $(71,85)$ a novembro $(70,69)$, o que pode ser visto na figura 3. Para segunda semana de idade, durante os meses de novembro $(70,69)$ a abril $(69,48)$, verificou-se que os valores estão dentro da faixa de conforto térmico, necessitando, portanto, de aquecimento entre os meses de maio $(64,56)$ a outubro $(68,09)$. Para a terceira semana de idade (ITU ideal 64,5 - 72), durante os meses de dezembro $(72,73)$ a fevereiro $(73,20)$, verificou-se a necessidade de um sistema de resfriamento, pois durante estes meses, os valores excederam o limite superior da faixa de conforto térmico para aves nesta idade, porém, entre os meses de março $(71,85)$ a novembro $(70,69)$, os valores se localizaram dentro da faixa de conforto térmico. Para a quarta semana de idade, entre os meses de outubro $(61,72)$ a maio $(64,56)$, necessitou-se de sistema de resfriamento do ambiente, pois apresentaram valores superiores ao máximo ideal, enquanto que, durante os meses de junho a $(61,72)$ a setembro $(64,98)$, os valores se localizaram dentro da faixa de conforto térmico para aves. Para sexta semana de idade, pôde-se verificar que durante todos os meses do ano, o município apresentou valores superiores ao máximo recomendado para aves, portanto, necessitando de sistema de resfriamento do ambiente, o que pode ser observado pela figura 4 . 
Tabela 3 - Índice de Temperatura e Umidade (ITU) para o município da Cerro Azul.

Table 3 - Temperature and humidity index (ITU) of Cerro Azul city

\begin{tabular}{|l|l|l|l|l|l|l|}
\hline & \multicolumn{6}{|c|}{ ITU } \\
\hline & \multicolumn{6}{|c|}{ Idade das Aves em Semanas } \\
\hline Meses do ano & $\mathbf{1}$ & $\mathbf{2}$ & $\mathbf{3}$ & $\mathbf{4}$ & $\mathbf{5}$ & $\mathbf{6}$ \\
\hline Janeiro & $\mathrm{C}$ & $\mathrm{C}$ & $\mathrm{S}$ & $\mathrm{S}$ & $\mathrm{S}$ & $\mathrm{S}$ \\
Fevereiro & $\mathrm{C}$ & $\mathrm{C}$ & $\mathrm{S}$ & $\mathrm{S}$ & $\mathrm{S}$ & $\mathrm{S}$ \\
Março & $\mathrm{I}$ & $\mathrm{C}$ & $\mathrm{C}$ & $\mathrm{S}$ & $\mathrm{S}$ & $\mathrm{S}$ \\
Abril & $\mathrm{I}$ & $\mathrm{C}$ & $\mathrm{C}$ & $\mathrm{S}$ & $\mathrm{S}$ & $\mathrm{S}$ \\
Maio & $\mathrm{I}$ & $\mathrm{I}$ & $\mathrm{C}$ & $\mathrm{C}$ & $\mathrm{S}$ & $\mathrm{S}$ \\
Junho & $\mathrm{I}$ & $\mathrm{I}$ & $\mathrm{I}$ & $\mathrm{C}$ & $\mathrm{C}$ & $\mathrm{S}$ \\
Julho & $\mathrm{I}$ & $\mathrm{I}$ & $\mathrm{I}$ & $\mathrm{C}$ & $\mathrm{C}$ & $\mathrm{S}$ \\
Agosto & $\mathrm{I}$ & $\mathrm{I}$ & $\mathrm{I}$ & $\mathrm{C}$ & $\mathrm{C}$ & $\mathrm{S}$ \\
Setembro & $\mathrm{I}$ & $\mathrm{I}$ & $\mathrm{C}$ & $\mathrm{C}$ & $\mathrm{C}$ & $\mathrm{S}$ \\
Outubro & $\mathrm{I}$ & $\mathrm{I}$ & $\mathrm{C}$ & $\mathrm{C}$ & $\mathrm{S}$ & $\mathrm{S}$ \\
Novembro & $\mathrm{I}$ & $\mathrm{C}$ & $\mathrm{C}$ & $\mathrm{S}$ & $\mathrm{S}$ & $\mathrm{S}$ \\
Dezembro & $\mathrm{C}$ & $\mathrm{C}$ & $\mathrm{S}$ & $\mathrm{S}$ & $\mathrm{S}$ & $\mathrm{S}$ \\
\hline
\end{tabular}

Figura 3 - Gráfico ITU obtido para aves na $1^{\text {a }}$ semana de idade em Cerro Azul. Figure 3 - ITU Graph for birds at first week of age in Cerro Azul.

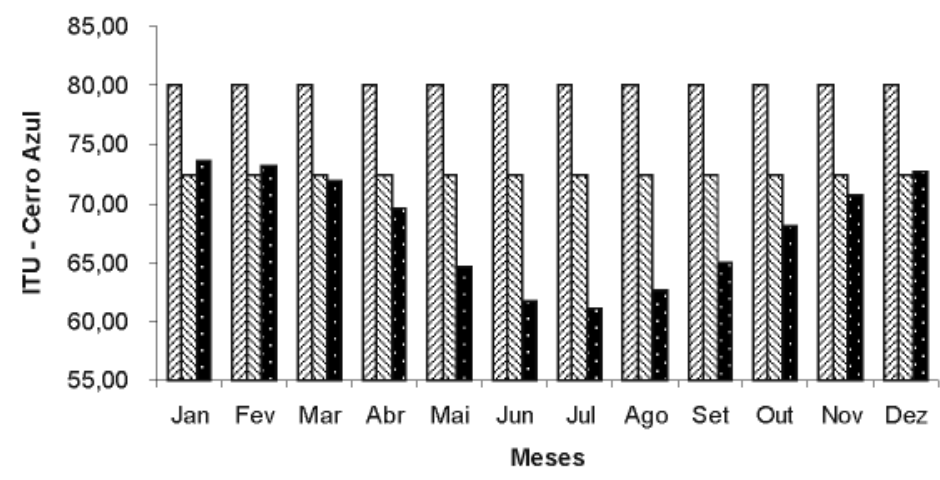

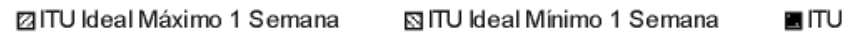

Figura 4 - Gráfico ITU obtido para aves na $6^{\text {a }}$ semana de idade em Cerro Azul. Figure 4 - ITU Graph for birds at sixty week of age in Cerro Azul.

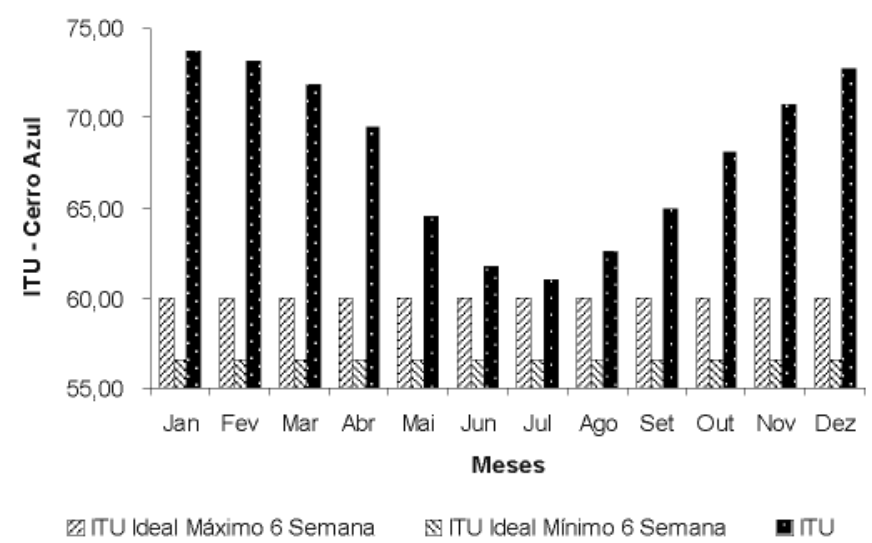


- Município Guaraqueçaba

Analisando-se as tabelas para ITU (TABELAS 1 e 4) nos meses de janeiro a dezembro, referente ao ciclo de vida de aves de corte, verificou-se que para a primeira semana de idade 0 valor encontrado para todos os meses do ano, no município de Guaraqueçaba, foi inferior ao ideal, de abril $(71,10)$ a novembro $(70,89)$, demonstrando a necessidade de sistema de aquecimento do ambiente para aves nesta faixa de idade, porém, durante os meses de dezembro $(73,11)$ a março $(73,47)$, os valores encontraram-se dentro da faixa de conforto térmico para aves, o que pode ser verificado pela figura 5. Na segunda semana de idade, os meses de novembro $(70,89)$ a abril $(71,10)$ apresentaram valores dentro da faixa de conforto térmico para aves, necessitando de aquecimento do ambiente a partir do mês de maio $(66,50)$ até outubro $(68,67)$. Para terceira semana de idade, foi necessário aquecimento do ambiente, entre os meses junho $(64,44)$ a agosto $(64,06)$, apresentando os meses de abril $(71,10)$, maio $(66,50)$, setembro $(65,50)$ a novembro $(70,98)$ valores dentro da faixa de conforto térmico e necessitando de aquecimento durante os meses de junho $(64,44)$ a agosto $(64,04)$. Para quarta semana de idade, os meses de outubro $(68,67)$ a abril $(71,10)$ apresentaram valores superiores ao ITU ideal, necessitando, portanto, de sistema de resfriamento com valores dentro da faixa de conforto térmico para os meses de maio $(66,50)$ a setembro $(65,50)$. Para a quinta semana de idade, apenas o mês de julho $(63,07)$ apresentou valor dentro da faixa de conforto térmico, no restante dos meses, fez-se necessária a utilização de um sistema de resfriamento. Para última semana, todos os meses excederam o limite superior, necessitando de um sistema de resfriamento, o que pode ser verificado na figura 6 .

Tabela 4 - Índice de Temperatura e Umidade (ITU) para o município da Guaraqueçaba. Table 4 - Temperature and Humidity Index (ITU) of Guaraqueçaba city.

\begin{tabular}{|l|l|l|l|l|l|l|}
\hline & \multicolumn{6}{|c|}{ ITU } \\
\hline & \multicolumn{7}{|c|}{ Idade das Aves em Semanas } \\
\hline Meses do ano & $\mathbf{1}$ & $\mathbf{2}$ & $\mathbf{3}$ & $\mathbf{4}$ & $\mathbf{5}$ & $\mathbf{6}$ \\
\hline Janeiro & $\mathrm{C}$ & $\mathrm{C}$ & $\mathrm{S}$ & $\mathrm{S}$ & $\mathrm{S}$ & $\mathrm{S}$ \\
Fevereiro & $\mathrm{C}$ & $\mathrm{C}$ & $\mathrm{S}$ & $\mathrm{S}$ & $\mathrm{S}$ & $\mathrm{S}$ \\
Março & $\mathrm{C}$ & $\mathrm{C}$ & $\mathrm{S}$ & $\mathrm{S}$ & $\mathrm{S}$ & $\mathrm{S}$ \\
Abril & $\mathrm{I}$ & $\mathrm{C}$ & $\mathrm{C}$ & $\mathrm{S}$ & $\mathrm{S}$ & $\mathrm{S}$ \\
Maio & $\mathrm{I}$ & $\mathrm{I}$ & $\mathrm{C}$ & $\mathrm{C}$ & $\mathrm{S}$ & $\mathrm{S}$ \\
Junho & $\mathrm{I}$ & $\mathrm{I}$ & $\mathrm{I}$ & $\mathrm{C}$ & $\mathrm{S}$ & $\mathrm{S}$ \\
Julho & $\mathrm{I}$ & $\mathrm{I}$ & $\mathrm{I}$ & $\mathrm{C}$ & $\mathrm{C}$ & $\mathrm{S}$ \\
Agosto & $\mathrm{I}$ & $\mathrm{I}$ & $\mathrm{I}$ & $\mathrm{C}$ & $\mathrm{S}$ & $\mathrm{S}$ \\
Setembro & $\mathrm{I}$ & $\mathrm{I}$ & $\mathrm{C}$ & $\mathrm{C}$ & $\mathrm{S}$ & $\mathrm{S}$ \\
Outubro & $\mathrm{I}$ & $\mathrm{I}$ & $\mathrm{C}$ & $\mathrm{S}$ & $\mathrm{S}$ & $\mathrm{S}$ \\
Novembro & $\mathrm{I}$ & $\mathrm{C}$ & $\mathrm{C}$ & $\mathrm{S}$ & $\mathrm{S}$ & $\mathrm{S}$ \\
Dezembro & $\mathrm{C}$ & $\mathrm{C}$ & $\mathrm{S}$ & $\mathrm{S}$ & $\mathrm{S}$ & $\mathrm{S}$ \\
\hline
\end{tabular}


Figura 5 - Gráfico ITU obtido para aves na $1^{\text {a }}$ semana de idade em Guaraqueçaba.

Figure 5 - ITU Graph for birds at first week of age in Guaraqueçaba.

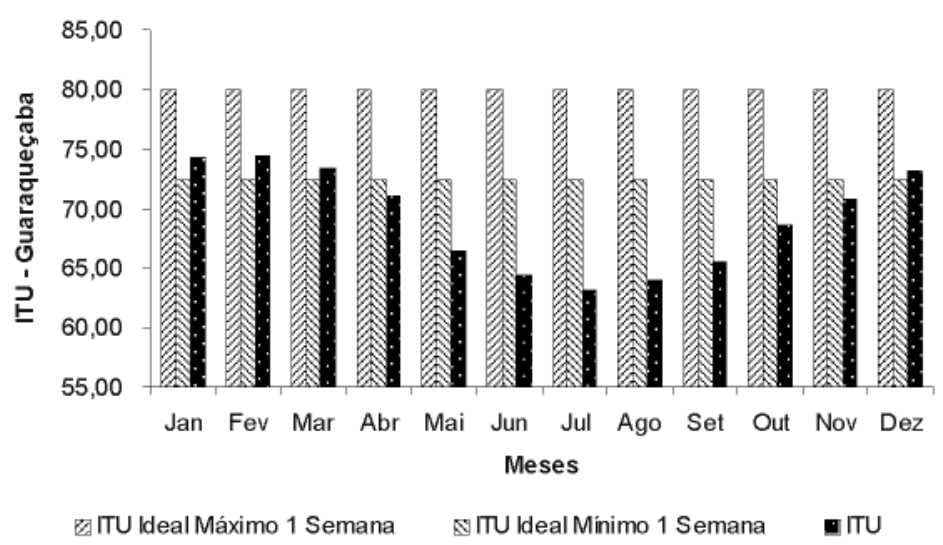

Figura 6 - Gráfico ITU obtido para aves na $6^{\text {a }}$ semana de idade em Guaraqueçaba. Figure 6 - ITU Graph for birds at sixty week of age in Guaraqueçaba.

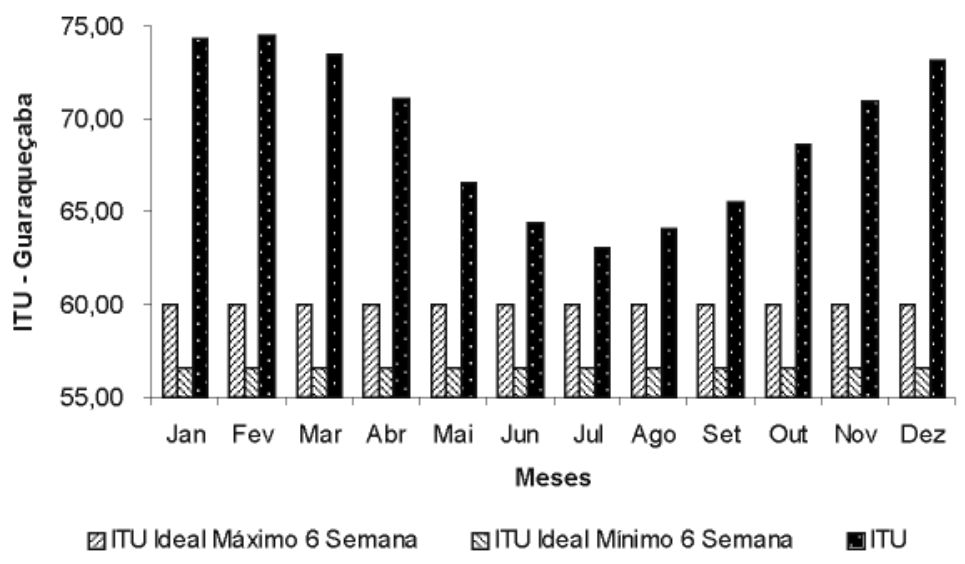

\section{- Município Lapa}

Analisando as tabelas para ITU (TABELAS 1 e 5) nos meses de janeiro a dezembro, referente ao ciclo de vida de aves de corte, verificou-se que para a primeira semana de idade, 0 valor encontrado para todos os meses do ano no município da Lapa é inferior ao ideal, portanto, há necessidade de sistema de aquecimento do ambiente para aves nesta faixa de idade, o que pode ser verificado pela figura 7 . Na segunda semana de idade, os meses de janeiro $(68,58)$ e fevereiro $(68,42)$ apresentaram valores dentro da faixa de conforto térmico para aves, necessitando de aquecimento do ambiente a partir do mês de março $(67,26)$ até dezembro $(67,57)$. Para ter- ceira semana de idade, foi necessário aquecimento do ambiente entre os meses maio $(60,01)$ a outubro $(63,41)$, apresentando os meses de novembro a abril valores dentro da faixa de conforto térmico. Para quarta semana de idade, os meses de janeiro $(68,58)$ e fevereiro $(68,42)$ apresentaram valores superiores ao ITU ideal, necessitando, portanto, de sistema de resfriamento, com valores dentro da faixa de conforto térmico para os meses de março $(67,26)$, abril $(64,55)$, outubro $(63,41)$, novembro $(65,38)$ e dezembro $(67,57)$; entre os meses de maio até setembro necessitouse de aquecimento do ambiente. Para a quinta semana de idade, os meses entre maio $(60,01)$ outubro $(63,41)$ apresentaram valores dentro da faixa de conforto térmico, porém, no restante dos 
meses, fez-se necessária a utilização de um sistema de resfriamento. Para última semana, apenas durante os meses de junho $(58,70)$, julho $(57,40)$ e agosto $(59,15)$ obtiveram-se valores dentro da faixa de conforto térmico, no restante dos meses os valores excederam o limite superior, necessitando de um sistema de resfriamento, o que pode ser verificado na figura 8.

Tabela 5 - Índice de Temperatura e Umidade (ITU) para o município da Lapa. Table 5 - Temperature and Humidity Index (ITU) of Lapa city.

\begin{tabular}{|l|l|l|l|l|l|l|}
\hline & \multicolumn{6}{|c|}{ ITU } \\
\hline & \multicolumn{7}{|c|}{ Idade das Aves em Semanas } \\
\hline Meses do ano & $\mathbf{1}$ & $\mathbf{2}$ & $\mathbf{3}$ & $\mathbf{4}$ & $\mathbf{5}$ & $\mathbf{6}$ \\
\hline Janeiro & I & C & C & S & S & S \\
Fevereiro & I & C & C & S & S & S \\
Março & I & I & C & C & S & S \\
Abril & I & I & C & C & S & S \\
Maio & I & I & I & I & C & S \\
Junho & I & I & I & I & C & C \\
Julho & I & I & I & I & C & C \\
Agosto & I & I & I & I & C & C \\
Setembro & I & I & I & I & C & S \\
Outubro & I & I & I & C & C & S \\
Novembro & I & I & C & C & S & S \\
Dezembro & I & I & C & C & S & S \\
\hline
\end{tabular}

Figura 7 - Gráfico ITU obtido para aves na $1^{\text {a }}$ semana de idade em Lapa.

Figure 7 - ITU Graph for birds at first week of age in Lapa.

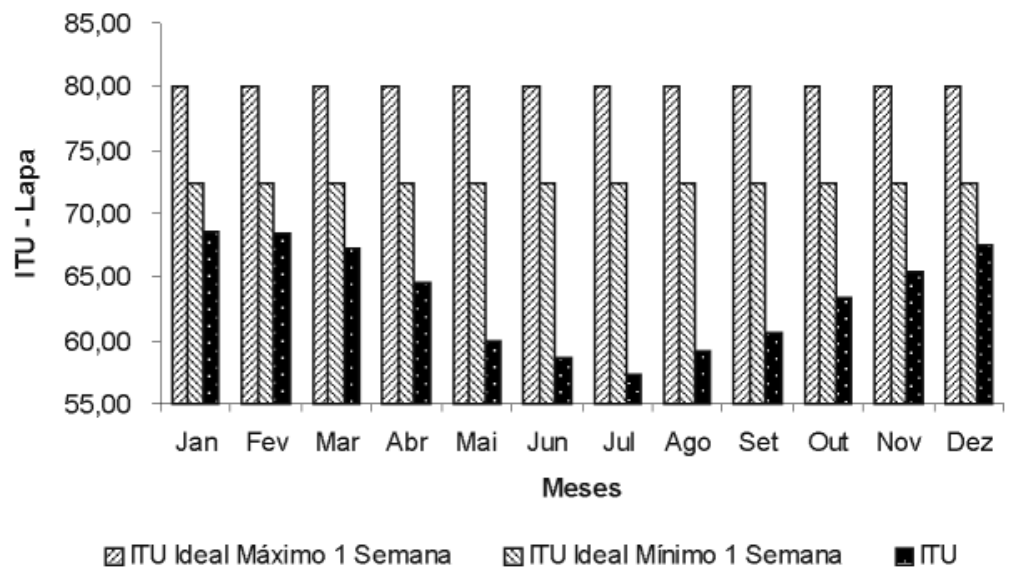




\section{Figura 8 - Gráfico ITU obtido para aves na $\boldsymbol{6}^{\mathrm{a}}$ semana de idade em Lapa.}

Figure 8 - ITU Graph for birds at sixty week of age in Lapa.

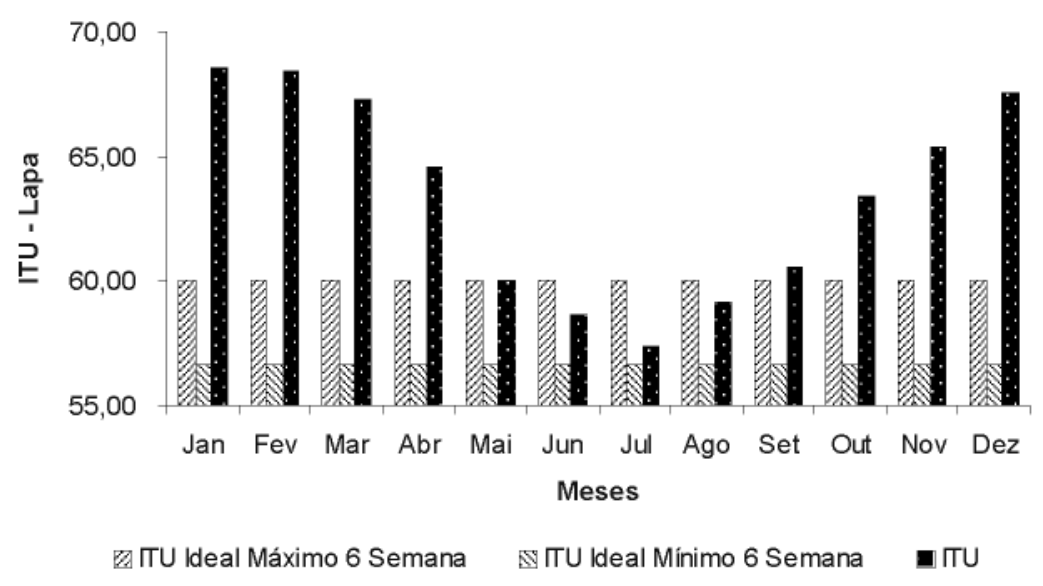

\section{- Município Morretes}

Analisando as tabelas para ITU (TABELAS 1 e 6) nos meses de janeiro a dezembro, referente ao ciclo de vida de aves de corte, verificou-se que para a primeira semana de idade, 0 valor encontrado para os meses de abril $(70,85)$ a novembro $(70,75)$, no município de Morretes, foi inferior ao ideal, portanto, necessitando do sistema de aquecimento do ambiente para aves nesta faixa de idade. Porém, durante os meses de dezembro $(73,00)$ a março $(73,06)$, os valores encontraram-se dentro da faixa de conforto térmi$\mathrm{co}, \mathrm{o}$ que pode ser verificado pela figura $9 . \mathrm{Na}$ segunda semana de idade, os meses de novembro $(70,75)$ a abril $(70,85)$ apresentaram valores dentro da faixa de conforto térmico para aves, necessitando de aquecimento do ambiente a partir do mês de maio $(66,49)$ até outubro $(68,31)$. Para terceira semana de idade, será necessário aquecimento do ambiente entre os meses junho $(64,01)$ a agosto $(63,78)$, apresentando os meses de abril, maio, setembro, outubro e novembro valores dentro da faixa de conforto térmico, porém durante os meses de novembro $(70,75)$ a março $(73,06)$, necessitando de sistemas de resfriamento. Para quarta semana de idade, os meses de novembro $(70,75)$ a abril $(70,85)$ apresentam valores superiores ao ITU ideal, necessitando, portanto, de sistema de resfriamento, valores dentro da faixa de conforto térmico para os meses de maio $(66,49)$ a outubro $(68,31)$. Para a quinta semana de idade, os meses junho $(64,01)$ a agosto $(63,78)$ apresentaram valores dentro da faixa de conforto térmico, porém no restante dos meses fez-se necessária a utilização de um sistema de resfriamento. Para última semana, em todos os meses os valores excederam o limite superior, necessitando de sistema de resfriamento, o que pode ser verificado na figura 10. 
Tabela 6 - Índice de Temperatura e Umidade (ITU) para Morretes.

Table 6 - Temperature and Humidity Index (ITU) of Morretes city.

\begin{tabular}{|l|l|l|l|l|l|l|}
\hline & \multicolumn{6}{|c|}{ ITU } \\
\hline & \multicolumn{7}{|c|}{ Idade das Aves em Semanas } \\
\hline Meses do ano & $\mathbf{1}$ & $\mathbf{2}$ & $\mathbf{3}$ & $\mathbf{4}$ & $\mathbf{5}$ & $\mathbf{6}$ \\
\hline Janeiro & $\mathrm{C}$ & $\mathrm{C}$ & $\mathrm{S}$ & $\mathrm{S}$ & $\mathrm{S}$ & $\mathrm{S}$ \\
Fevereiro & $\mathrm{C}$ & $\mathrm{C}$ & $\mathrm{S}$ & $\mathrm{S}$ & $\mathrm{S}$ & $\mathrm{S}$ \\
Março & $\mathrm{C}$ & $\mathrm{C}$ & $\mathrm{S}$ & $\mathrm{S}$ & $\mathrm{S}$ & $\mathrm{S}$ \\
Abril & $\mathrm{I}$ & $\mathrm{C}$ & $\mathrm{C}$ & $\mathrm{S}$ & $\mathrm{S}$ & $\mathrm{S}$ \\
Maio & $\mathrm{I}$ & $\mathrm{I}$ & $\mathrm{C}$ & $\mathrm{C}$ & $\mathrm{S}$ & $\mathrm{S}$ \\
Junho & $\mathrm{I}$ & $\mathrm{I}$ & $\mathrm{I}$ & $\mathrm{C}$ & $\mathrm{C}$ & $\mathrm{S}$ \\
Julho & $\mathrm{I}$ & $\mathrm{I}$ & $\mathrm{I}$ & $\mathrm{C}$ & $\mathrm{C}$ & $\mathrm{S}$ \\
Agosto & $\mathrm{I}$ & $\mathrm{I}$ & $\mathrm{I}$ & $\mathrm{C}$ & $\mathrm{C}$ & $\mathrm{S}$ \\
Setembro & $\mathrm{I}$ & $\mathrm{I}$ & $\mathrm{C}$ & $\mathrm{C}$ & $\mathrm{S}$ & $\mathrm{S}$ \\
Outubro & $\mathrm{I}$ & $\mathrm{I}$ & $\mathrm{C}$ & $\mathrm{C}$ & $\mathrm{S}$ & $\mathrm{S}$ \\
Novembro & $\mathrm{I}$ & $\mathrm{C}$ & $\mathrm{C}$ & $\mathrm{S}$ & $\mathrm{S}$ & $\mathrm{S}$ \\
Dezembro & $\mathrm{C}$ & $\mathrm{C}$ & $\mathrm{S}$ & $\mathrm{S}$ & $\mathrm{S}$ & $\mathrm{S}$ \\
\hline
\end{tabular}

Figura 9 - Gráfico ITU obtido para aves na $1^{\text {a }}$ semana de idade em Morretes. Figure 9 - ITU Graph for birds at first week of age in Morretes.

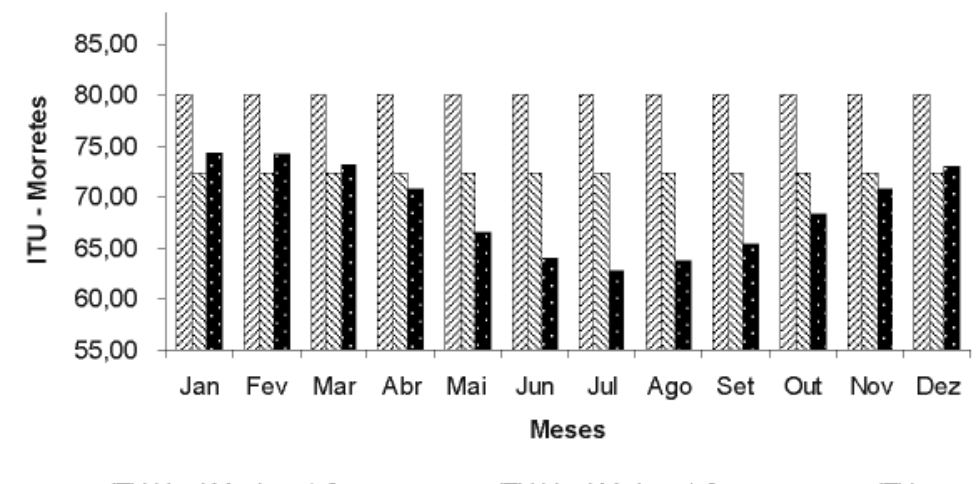

圆 ITU Ideal Máximo 1 Semana $\quad$ ITU Ideal Mínimo 1 Semana ㅁ ITU

Figura 10 - Gráfico ITU obtido para aves na $6^{\text {a }}$ semana de idade em Morretes.

Figure 10 - ITU Graph for birds at sixty week of age in Morretes.

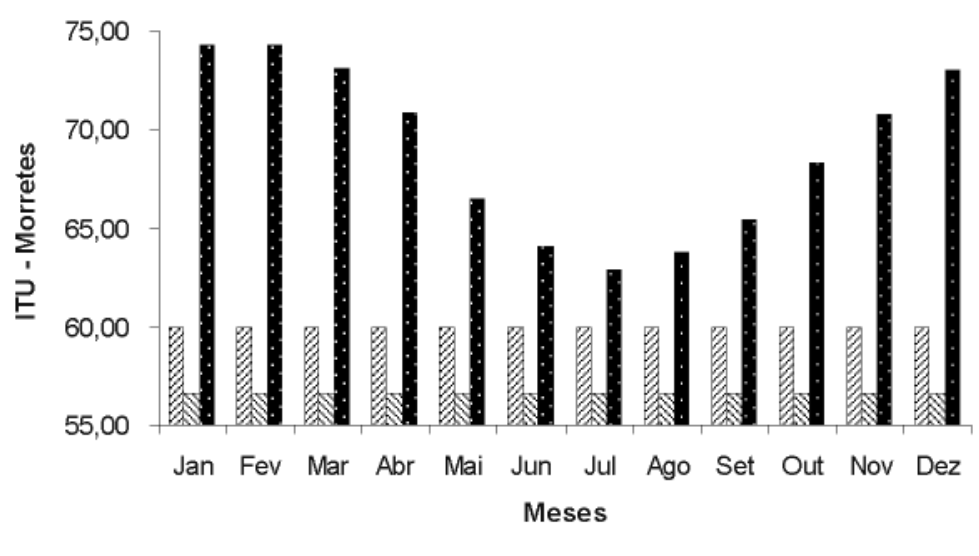

囷 TU Ideal Máximo 6 Semana $\mathbb{N} \pi U$ Ideal Mínimo 6 Semana 


\section{- Município Pinhais}

Analisando as tabelas para ITU (tabelas 1 e 7), nos meses de janeiro a dezembro, referente ao ciclo de vida de aves de corte, verificou-se que para a primeira semana de idade, o valor encontrado para todos os meses do ano no município de Pinhais é inferior ao ideal, portanto, existiu necessidade de sistema de aquecimento do ambiente para aves nesta faixa de idade, 0 que pode ser verificado pela figura 11. Na segunda semana de idade, os meses de janeiro $(68,53)$ e fevereiro $(68,18)$ apresentaram valores dentro da faixa de conforto térmico para aves, necessitando de aquecimento do ambiente a partir do mês de março $(66,85)$ até dezembro $(67,16)$. Para terceira semana de idade, foi necessário aquecimento do ambiente entre os meses maio $(60,30)$ a outubro $(62,93)$, apresentando os meses de novembro $(65,21)$ a abril $(64,64)$ valores dentro da faixa de conforto térmico. Para quarta semana de idade, os meses de janeiro $(68,53)$ e fevereiro $(68,18)$ apresentaram valores superiores ao ITU ideal, necessitando, portanto, de sistema de resfriamento; a partir do mês de maio $(60,30)$ a setembro $(60,36)$, necessitou-se de aquecimento do ambiente. Para a quinta semana de idade, os meses entre maio $(60,30)$ e outubro $(62,93)$ apresentaram valores dentro da faixa de conforto térmico, porém, no restante dos meses, fez-se necessária a utilização de um sistema de resfriamento. Para última semana, apenas durante os meses entre junho $(58,17)$, julho $(57,77)$ e agosto $(58,81)$ obtiveram-se valores dentro da faixa de conforto térmico; no restante dos meses, os valores excederam o limite superior, necessitando de um sistema de resfriamento, o que pode ser verificado na figura 12.

Tabela 7 - Índice de Temperatura e Umidade (ITU) para Pinhais.

Table 7 - Temperature and humidity Index (ITU) of Pinhais city.

\begin{tabular}{|l|l|l|l|l|l|l|}
\hline & \multicolumn{6}{|c|}{ ITU } \\
\hline & \multicolumn{7}{|c|}{ Idade das Aves em Semanas } \\
\hline Meses do ano & $\mathbf{1}$ & $\mathbf{2}$ & $\mathbf{3}$ & $\mathbf{4}$ & $\mathbf{5}$ & $\mathbf{6}$ \\
\hline Janeiro & I & C & C & S & S & S \\
Fevereiro & I & C & C & S & S & S \\
Março & I & I & C & C & S & S \\
Abril & I & I & C & C & S & S \\
Maio & I & I & I & I & C & S \\
Junho & I & I & I & I & C & C \\
Julho & I & I & I & I & C & C \\
Agosto & I & I & I & I & C & C \\
Setembro & I & I & I & C & C & S \\
Outubro & I & I & I & C & C & S \\
Novembro & I & I & C & C & S & S \\
Dezembro & I & I & C & C & S & S \\
\hline
\end{tabular}




\section{Figura 11 - Gráfico ITU obtido para aves na $1^{\text {a }}$ semana de idade em Pinhais.}

Figure 11 - ITU Graph for birds at first week of age in Pinhais.

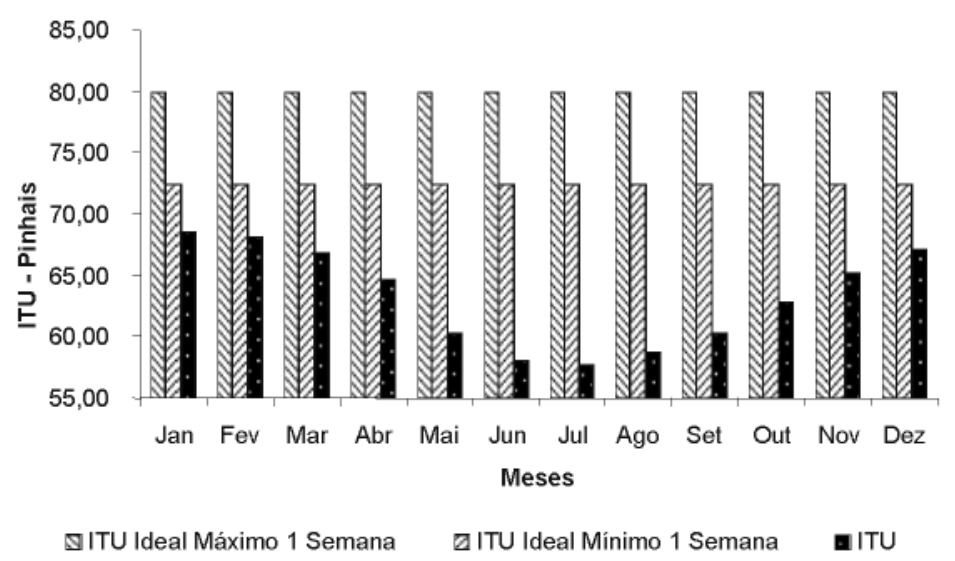

Figura 12 - Gráfico ITU obtido para aves na $6^{\text {a }}$ semana de idade em Pinhais. Figure 12 - ITU Graph for birds at sixty week of age in Pinhais.

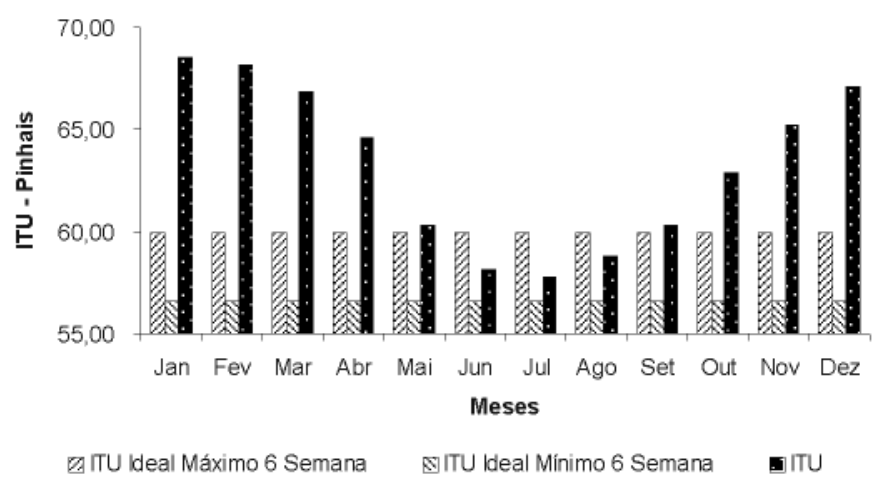

\section{Conclusões}

- A maioria das microrregiões não apresentou condições para um ótimo desenvolvimento das aves;

- O ITU mostra a necessidade de correção do microclima para se obter condições ideais de conforto térmico para a produção de aves;
- É necessária a realização de um estudo da viabilidade econômica, levando-se em consideração os investimentos necessários para obter condições necessárias de conforto térmico para produção de aves. 


\section{Referências}

MULLER, P. B. Bioclimatologia aplicada aos animais domésticos. Porto Alegre: Sulina, 1989. P. 262.

PIASENTINI, J. A. Conforto medido pelo índice de temperatura do globo e umidade na produção de frangos de corte para dois tipos de pisos em Viçosa, MG. 1958. $77 \mathrm{f}$. Dissertação (Mestrado) - UFV, 1958.
THOM, E. C. Cooling degree-days air conditioning, heating and ventilating. Atlanta, GA, USA. Transactions of ASH\&VE. v. 55, p. 65-72, 1958.

TINÔCO, I. F. F., Ambiência e instalações para avicultura industrial. CONGRESSO BRASILEIRO DE ENGENHARIA AGRÍCOLA, 27, Poços de Caldas, MG, Anais..., Terceiro Encontro Nacional de Técnicos, Pesquisadores e Educadores de construções Rurais. Editado por Victor Hugo Teixeira, Lúcia Ferreira. Lavras: UFLA/SBEA, 1998. p. 1-86. 